\title{
Superior Ophthalmic Vein Thrombosis Presenting with Headaches and Diplopia
}

\author{
Maryam Naser MD*, Nafiseh Hashemi MD and Ashkan Mowla MD
}

*Corresponding author: Maryam Naser MD, Neuro-ophthalmology Clinic, Hashemi Eye Care, Encino, USA.

\begin{abstract}
Superior ophthalmic vein thrombosis (SOVT) is an extremely rare orbital pathology which is usually associated with orbital inflammation and infection, hypercoagulable states, autoimmune conditions, tumor compression, carotid cavernous fistula (CCF), vascular malformation and trauma [1-4]. Clinical findings include sudden onset of painful proptosis, ophthalmoplegia, chemosis and visual disturbance. SOVT can be life threatening due to risk of cavernous sinus thrombosis. Early diagnosis is important and appropriate management based on the underlying causative factors is essential to prevent complications like vision loss [5]. We present a case of superior ophthalmic vein thrombosis of the right eye.
\end{abstract}

Keywords: Cavernous sinus thrombosis; Proptosis; Superior ophthalmic vein thrombosis

\section{Case Presentation}

A 68-year-old white female with past medical history of coronary artery disease, myocardial infraction, aneurysm clipping, cholecystectomy, myasthenia gravis, migraine and anxiety presented with worsening of headache. The patient complained of increased in intensity of her headaches to 10 out of 10 . The pain was throbbing, intermittent and localized behind right eye. Headaches was associated with photophobia and intermittent diplopia. MRI brain and orbit showed possible venous thrombosis. MRA verified thrombosis in right superior ophthalmic vein. The medications she used to take at home were Lasix $40 \mathrm{mg}$, nitroglycerin $0.4 \mathrm{mg}$ sublingual PRN, Ativan $1 \mathrm{mg}$ TID. On review of system, there was no history of fever or chills change in appetite, hearing change or ear discharge, sinus disease. Vitals were normal except for HR of 108.

On physical examination, pupils were equal and reactive to light ( $4 \mathrm{~mm}$ in dark, $2 \mathrm{~mm}$ in light). Extra ocular movements were intact. She had binocular diplopia and proptosis of right eye. Rest of the neurological exam was normal. Hematological workup was negative for hypercoagulability state. The patient was started on heparin per stroke protocol with recommendation of discharging on Coumadin. Gradual resolution of symptoms was noted after 4 day (Figure $1 \& 2$ ).
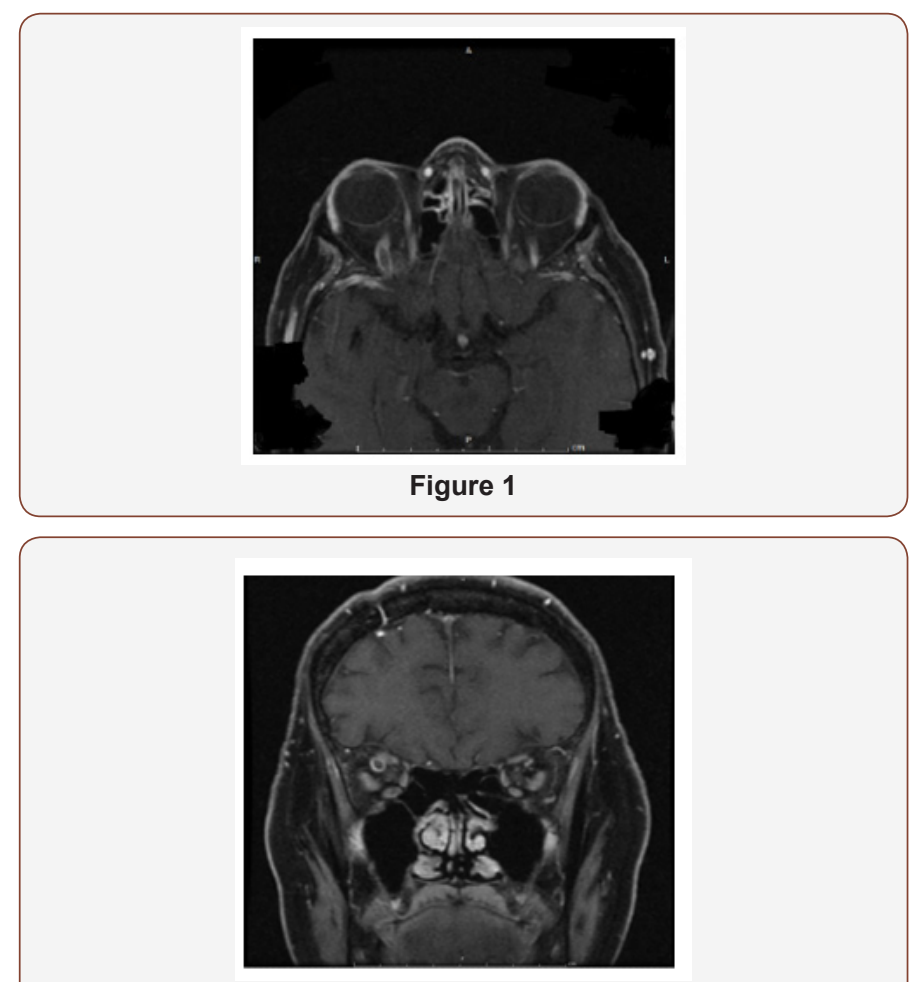

Figure 2 


\section{Discussion}

Superior Ophthalmic Vein Thrombosis (SOVT) is a rare entity characterized by rapidly progressive symptoms including ophthalmoplegia, periorbital edema and proptosis due to blockage of venous drainage from the orbit $[3,6]$. In a retrospective case series of three patients with confirmed SOVT, all patients presented with unilateral, painful proptosis noted upon awakening [3]. Although the etiology of SOVT is multifactorial, usual factors include coagulation disorders, vascular and Sino orbital disease. The most common cause of SOVT is orbital infection, as in the case of orbital cellulitis [7]. Other etiologies including vascular malformations, thyroid associated orbitopathy, and sarcoidosis also have been reported.

SOVT may be challenging to differentiate between orbital cellulitis, because of the overlap of symptoms and similar clinical presentation [8]. In SOVT, fundoscopic examination is normal [1]. The exact pathogenesis of SOVT is unknown, however systemic infection may lead to a procoagulant response. Paranasal sinusitis may lead to congestion of SOV and ultimately thrombus formation. Diagnosis by imaging modalities may be challenging. Enhanced CT shows dilation of SOV accompanied by peripheral enhancement and central hypo intensity [8].

However, it may not reveal early thrombosis or early cavernous sinus thrombosis. Due to these reasons, authors believe that MRI is a more sensitive and specific tool [1]. MRI and MRV can confirm the diagnosis of SOVT and exclude other mimicking disorders such as cavernous sinus thrombosis and Sino-orbital infection [3]. The absence of expected signal void in the SOV is a sign of thrombosis [9]. A case review pointed to three reported cases of SOVT with restricted diffusion, reflecting the presence of an intraventricular clot [6]. On cerebral angiography, SOVT is demonstrated by thickening of the vessel lumen [1]. Treatment is guided by consideration of severity of findings. Anticoagulation is controversial and should be considered after risk and benefit analysis [3]. Early detection of SOVT is important in avoiding cavernous sinus thrombosis [9]. In patients with ophthalmoplegia and periorbital edema, the diagnosis of SOVT should be considered to prevent potentially serious complications.

\section{Conclusion}

SOVT is an early sign of impending cavernous sinus thrombosis which could be life threatening. Anticoagulants may be considered in such patients. A multidisciplinary approach with the treating physician, neurologist, radiologist and ophthalmologist is suggested when managing a case of orbital vein thrombosis. Risk factors for spontaneous superior ophthalmic vein thrombosis are multifactorial. MRI and MRV is the imaging modality of choice for confirming SOVT and can help to exclude other disorders which may mimic the condition.

\section{Acknowledgement}

None.

\section{Conflicts of Interest}

No conflict of interest.

\section{References}

1. Cumurcu T, Demirel S, Keser S, Bulut T, Cavdar M, et al. (2013) Superior ophthalmic vein thrombosis developed after orbital cellulitis. Semin Ophthalmol 28(2): 58-60.

2. Mandić JJ, Mandić K, Mrazovac D (2018) Superior ophthalmic vein thrombosis with complete loss of vision as a complication of autoimmune and infective conditions. Ocul Immunol Inflamm 26(7): 1066-1068.

3. Lim LH, Scawn RL, Whipple KM, Oh SR, Lucarelli MJ, et al. (2014) Spontaneous superior ophthalmic vein thrombosis: A rare entity with potentially devastating consequences. Eye (Lond) 28(3): 348-351.

4. Michaelides M, Aclimandos W (2003) Bilateral superior ophthalmic vein thrombosis in a young woman. Acta Ophthalmol Scand 81(1): 88-90.

5. B Pawar, AM Matthews (2017) Superior Ophthalmic Vein Thrombosis Associated with Orbital Inflammation. Adv Ophthalmol Vis Syst 7(2): 216.

6. Choi JH, Jung JH, Baik SK, Park KP, Choi KD (2014) Restricted diffusion in isolated superior ophthalmic vein thrombosis. J Neuroophthalmol 34(1): 98-99.

7. Kim SC, Cho SY (2013) Superior ophthalmic vein thrombosis in a patient with adenoid cystic carcinoma in middle meatus. Can J Ophthalmol 48(1): e12-14.

8. Akiyama K, Karaki M, Samukawa Y, Mori N (2013) Blindness caused by septic superior ophthalmic vein thrombosis in a Lemierre Syndrome variant. Auris Nasus Larynx 40(5): 493-496.

9. Coban G, Cetinkaya A, Karalezli A, Donmez FY, Ozbek N (2013) Unilateral superior ophthalmic vein thrombosis in a neonate. Ophthalmic Plast Reconstr Surg 29(6): e154-156. 